\title{
Coupled numerical simulation of multi-layer reservoir developed by lean-stratified water injection
}

\author{
Dapeng Gao ${ }^{1,2,3} \cdot$ Jigen $\mathrm{Ye}^{2} \cdot$ Yuewu Liu ${ }^{1}$ Songqi Pan ${ }^{2,3} \cdot$ Yunpeng $\mathrm{Hu}^{2} \cdot$ \\ He Yuan ${ }^{2}$
}

Received: 5 July 2015/Accepted: 13 February 2016/Published online: 26 February 2016

(C) The Author(s) 2016. This article is published with open access at Springerlink.com

\begin{abstract}
Lean-stratified water injection is one of the most important technologies to increase production and develop potentials for the oilfield with extreme high water content. However, traditional models cannot entirely solve the inner boundary conditions of lean-stratified water injection. Therefore, we established the injection wellbore constraint equations, which were coupled with the oil/water two-phase numerical reservoir models, and then the seven diagonal form sparse coefficient matrix was solved by block precondition of generalized minimal residual algorithm. Considering the specific situation of lean-stratified water injection wells, reservoir geology and production schemes of the middle part of the sixth Oilfield in Xing Shugang, three mechanism models of multi-layer heterogeneous reservoir were constructed to simulate the leanstratified water injection. The influences of different segments numbers, modes of combination in segment layer and rhythm characteristics of remaining oil reserves and distribution are evaluated.
\end{abstract}

Keywords Lean-stratified water injection - Well model · Numerical reservoir simulation - Remaining oil evaluation · Interactive low-permeability reservoir

Dapeng Gao

gaodapeng2009@163.com

1 Institute of Mechanics, Chinese Academy of Sciences, Beijing 100190, China

2 PetroChina Research Institute of Petroleum Exploration and Development, Beijing 100083, China

3 School of Earth and Space Science, Peking University, Beijing 100871, China

\section{Introduction}

Lean-stratified water injection (LSWI) is a kind of layer targeted water injection to enhance waterflooding recovery of multi-layer reservoirs, which divides wellbore into five or more segments, and every segment injects water for less than six layers. This technology can improve waterflood vertical sweep efficiency, and has been widely used in many multilayer sandstone reservoirs with high water content $(\mathrm{He}$ et al. 2013), such as Chinese Daqing Oilfield with higher than $40 \%$ waterflood recovery. Furthermore, it is required to match the development dynamic history for every oil layer in the process of reservoir numerical simulation during secondary development, for the sake of increasing the prediction precision for remaining oil in each sand body, especially in main sand body (Dakuang 2010).

The water injection rate enters each oil layer cannot follow the mobility allocated principle set in conventional simulation model. This defect leads to inaccuracy for matching the remaining oil distribution and injected water rate in reservoir numerical simulation. Reservoir numerical simulation methods considering LSWI contain injected water deduplication by many injection wells and coupled simulation for water chokes, injection well and reservoir. The former is suitable for reservoir possessed enough injectivity test data, and the water injection rate can be calculated by real-time monitoring test data, and these values can be used as inner boundary condition in model (Dumkwu et al. 2012; Villanueva-Triana et al. 2013; Stone et al. 1989; Ding 2011), practically, injectivity test data are very few, so the complete injection process cannot be represented by them (Gao et al. 2015). The latter needs enough data of injection well devices, such as diameters of water chokes, which should be used to calculate the pressure loss coupled with well bottom pressure (BHP) and

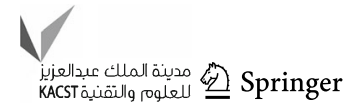


reservoir pressure (Zhao et al. 2012; Lin et al. 2011), and this results in quit complex calculation. In this paper, we propose a simple method of modeling LSWI considering inner wellbore constraint condition, and this method can be used to simulate the development effect and remaining oil distribution in reservoir with extreme high water content, so a reliable evidence will be provided for reservoir history matching and project design.

\section{Well model considering LSWI}

A new well model considering LSWI should be constructed as the inner boundary condition for numerical reservoir simulation, and the following assumptions are made.

1. Injection well is divided into $N$ segments vertically, and every segment contains $M$ oil layers.

2. The total injected water rate is known, and maintains constant.

3. Every segment is separated by packers in tubingcasing annulus, so there is no fluid flowing through between them.

4. Only oil and water phases are considered in the model, and the capillary is neglected.

The total injected water rate is expressed as:

$Q_{\mathrm{w}}=\sum_{\mathrm{seg}=1}^{N} Q_{\mathrm{w}_{(\mathrm{seg})}}=\sum_{\mathrm{seg}=1}^{N} \sum_{\text {lay }=1}^{M} q_{\mathrm{w}_{(\mathrm{seg})(\mathrm{lay})}}$

Every injection rate of each segment of wellbore is solved by linear implicit method to enhance the stability of numerical equations. Taking water saturation, injection pressure and oil phase pressure as unknown parameters, the injection rate can be expressed by Taylor-formula at two continuous time steps as Eq. 2.

$$
\begin{aligned}
& Q_{\mathrm{w}_{(\mathrm{seg})}}^{n+1} \approx Q_{\mathrm{w}_{(\mathrm{seg})}}^{n}
\end{aligned}
$$

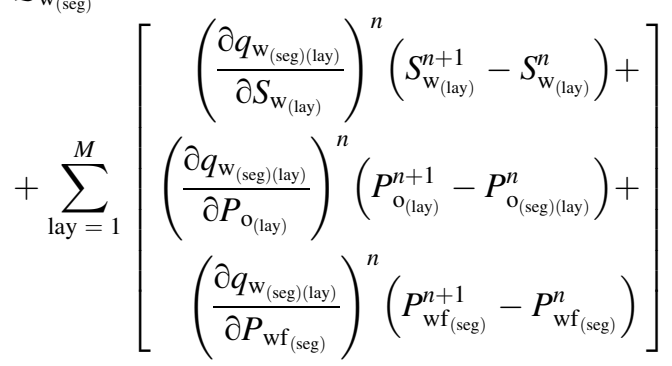

Although injected water rate of every oil layer varies during these two continuous time steps, the total injected water rate of every segment is constant $\left(Q_{w_{(\text {seg })}}^{n+1}=Q_{\mathrm{w}_{(\mathrm{seg})}}^{n}\right)$ under the assumption above, and then the well model considering LSWI is deduced from Eq. 2.

$$
\begin{aligned}
P_{\mathrm{wf}_{(\mathrm{seg})}}^{n+1}= & P_{\mathrm{wf}_{\text {(seg) }}}^{n} \\
& -\sum_{\text {lay }=1}^{M}\left[\begin{array}{l}
\left(\frac{\partial q_{\mathrm{w}_{(\text {seg }) \text { (lay) }}}}{\partial S_{\mathrm{w}_{(\text {lay })}}}\right)^{n}\left(S_{\mathrm{w}_{(\text {lay })}}^{n+1}-S_{\mathrm{w}_{(\text {lay })}}^{n}\right)+ \\
\left(\frac{\partial q_{\mathrm{w}_{(\text {seg })(\text { lay })}}}{\partial P_{\mathrm{o}_{(\text {lay })}}}\right)^{n}\left(P_{\mathrm{o}_{(\text {lay })}}^{n+1}-P_{\mathrm{o}_{(\text {lay })}}^{n}\right.
\end{array}\right]
\end{aligned}
$$$$
/ \sum_{\text {lay }=1}^{M}\left(\frac{\partial q_{\mathrm{w}_{(\mathrm{seg})(\mathrm{lay})}}}{\left.\partial P_{\mathrm{wf}}\right)_{(\mathrm{seg})}}\right)^{n}
$$

where $Q_{\mathrm{w}}$ is the total injected rate; seg is the label for segment; lay is the label for layer; $n$ is the label for time step; $N$ is the total segment number for a LSWI well; $M$ is the total number of layers; $q_{\mathrm{w}}$ is the injected water rate for every oil layer; $s_{\mathrm{W}}$ is the water saturation; $P_{\mathrm{o}}$ is oil pressure; $P_{\mathrm{wf}}$ is injected pressure adjusted by water chokes.

\section{Coupled numerical model}

Assuming that the pressure gradient in tubing-casing annulus is constant at different time steps, and then the injected pressure after water chokes and connected reservoir grid flow rate and pressure are given by:

Wellbore pressure model:

$P_{\mathrm{wf}_{a}}^{n+1}=P_{\mathrm{wf}_{(\mathrm{seg})}^{n+1}}^{+}+\xi_{\mathrm{w}}\left(H_{a}-H_{(\mathrm{seg})}\right)$

Flow model of reservoir grid (Yan et al. 2008):

$q_{\mathrm{w}_{a}}^{n+1}=q_{w_{(\mathrm{seg})(\mathrm{lay})}}^{n+1}=\mathrm{WI}_{\mathrm{w}_{a}}\left(P_{\mathrm{wf}_{a}}^{n+1}-P_{\mathrm{o}_{a}}^{n+1}-\lambda \frac{L_{a}}{2}\right)$

Oil-water two phase flow models are discretized, and pressure and saturation are solved implicitly, residual equations of the reservoir grid with producer or injector contain source and sink terms.

Oil phase residual equation:

$$
\begin{aligned}
R_{\mathrm{o}_{a}}= & \frac{V_{a}}{\Delta t}\left[\left(\frac{\phi\left(1-S_{\mathrm{W}}\right)}{B_{\mathrm{o}}}\right)_{a}^{n+1}-\left(\frac{\phi\left(1-S_{\mathrm{W}}\right)}{B_{\mathrm{o}}}\right)_{a}^{n}\right] \\
& -\sum_{i=1}^{b}\left[T_{\mathrm{o}_{a i}}\left(\Delta P-\gamma_{\mathrm{o}} \Delta Z\right)\right]^{n+1}-q_{\mathrm{o}_{a}}^{n+1}
\end{aligned}
$$

Water phase residual equation:

$$
\begin{aligned}
R_{\mathrm{w}_{a}}= & \frac{V_{a}}{\Delta t}\left[\left(\frac{\phi S_{\mathrm{w}}}{B_{\mathrm{w}}}\right)_{a}^{n+1}-\left(\frac{\phi S_{\mathrm{w}}}{B_{\mathrm{w}}}\right)_{a}^{n}\right] \\
& -\sum_{i=1}^{b}\left[T_{\mathrm{w}_{a i}}\left(\Delta P-\gamma_{\mathrm{w}} \Delta Z\right)\right]^{n+1}-q_{\mathrm{w}_{a}}^{n+1}
\end{aligned}
$$


Oil phase residual equations of reservoir grid without producer and injector are conventional as heavy oil model, injected pressure needs to be considered only for these grids with producer and injector, and these terms are functions of water saturation and oil pressure as represented in Eq. 3, so the unknown parameters are water saturation and oil pressure when solving the nonlinear equation set consists of these residual equations of every grids. Coupled with well model, wellbore pressure model and reservoir grid flow model, water phase residual equation for reservoir grids with injector is expressed as:

$$
\left\{\begin{array}{l}
\left(\frac{\partial R_{\mathrm{o}_{a}}}{\partial P_{\mathrm{o}_{a}}}\right)^{n}\left(P_{\mathrm{o}_{a}}^{n+1}-P_{\mathrm{o}_{a}}^{n}\right)+\left(\frac{\partial R_{\mathrm{O}_{a}}}{\partial S_{\mathrm{w}_{a}}}\right)^{n}\left(S_{\mathrm{w}_{a}}^{n+1}-S_{\mathrm{w}_{a}}^{n}\right)=R_{\mathrm{o}_{a}} \\
\left(\frac{\partial R_{\mathrm{w}_{a}}}{\partial P_{\mathrm{o}_{a}}}\right)^{n}\left(P_{\mathrm{o}_{a}}^{n+1}-P_{\mathrm{o}_{a}}^{n}\right)+\left(\frac{\partial R_{\mathrm{w}_{a}}}{\partial S_{\mathrm{w}_{a}}}\right)^{n}\left(S_{\mathrm{w}_{a}}^{n+1}-S_{\mathrm{w}_{a}}^{n}\right)=R_{\mathrm{w}_{a}}
\end{array}\right.
$$

Every element in the coefficient matrix can be simplified as a $2 \times 2$ submatrix: $\left[\begin{array}{cc}A_{P_{\mathrm{o}}}^{\mathrm{o}} & A_{S_{\mathrm{w}}}^{\mathrm{o}} \\ A_{P_{\mathrm{o}}}^{\mathrm{w}} & A_{S_{\mathrm{w}}}^{\mathrm{w}}\end{array}\right]$, where $\xi_{\mathrm{w}}$ is water pressure gradient in well segment; $a$ is the label of center grid; $H$ is depth; $i$ is the adjacent grid; $\lambda$ is threshold pressure gradient; $L_{a}$ is the distance between well and grid boundary; $R_{\mathrm{O}_{a}}$ is the oil phase residual for center grid; $R_{\mathrm{w}_{a}}$ is the water phase residual for center grid; $b$ is the total number of adjacent grids; $T_{\mathrm{o}}, T_{\mathrm{w}}$ are the oil and water transmissibility between grids; $B_{\mathrm{o}}, B_{\mathrm{w}}$ are the oil and water volume factors; $q_{\mathrm{o}}, q_{\mathrm{w}}$ are the injected and produced oil/water rate in grid; WI is well index; $\gamma_{0}, \gamma_{w}$ are oil/water gravity; $\phi$ is the porosity.

All of the oil-water residual equations of reservoir grids are combined as a huge linear equation set (Eq. 9), which can also be written as the matrix formats with seven diagonal sparse coefficient matrix, and its structure is as same as the coefficient matrix of conventional reservoir numerical simulation model without LSWI (Jigen et al. 2007).

$$
\begin{aligned}
& A_{P_{\mathrm{o}}}^{\mathrm{w}}=\frac{\partial R_{\mathrm{w}_{a}}}{\partial P_{\mathrm{o}_{a}}}=\frac{V_{a}}{\Delta t} \frac{\partial\left(\frac{\phi S_{\mathrm{w}}}{B_{\mathrm{w}}}\right)_{a}^{n+1}}{\partial P_{\mathrm{o}_{a}}}-\frac{\partial\left\{\sum_{i=1}^{b}\left[T_{\mathrm{w}_{a i}}\left(\Delta P-\gamma_{\mathrm{w}} \Delta Z\right)\right]^{n+1}\right\}}{\partial P_{\mathrm{o}_{a}}} \\
& -\frac{\partial \mathrm{WI}_{\mathrm{w}_{a}}}{\partial P_{\mathrm{o}_{a}}}\left\{\begin{array}{c}
P_{w \mathrm{f}_{(\mathrm{seg})}}^{n}+\xi_{\mathrm{w}}\left(H_{a}-H_{(\mathrm{seg})}\right)-P_{\mathrm{o}_{a}}^{n+1}-\lambda \frac{L_{a}}{2} \\
-\frac{\sum_{\mathrm{lay}=1}^{M}\left[\begin{array}{r}
\left(\frac{\partial q_{\mathrm{w}_{a}}}{\partial S_{\mathrm{w}_{a}}}\right)^{n}\left(S_{\mathrm{w}_{a}}^{n+1}-S_{\mathrm{w}_{a}}^{n}\right) \\
+\left(\frac{\partial q_{\mathrm{w}_{a}}}{\partial P_{\mathrm{o}_{a}}}\right)^{n}\left(P_{\mathrm{o}_{a}}^{n+1}-P_{\mathrm{o}_{a}}^{n}\right)
\end{array}\right]}{\sum_{\mathrm{lay}=1}^{M}\left(\frac{\partial q_{\mathrm{w}_{a}}}{\partial P_{\mathrm{wf}_{(\mathrm{seg})}}}\right)^{n}}
\end{array}\right\} \\
& +\mathrm{WI}_{\mathrm{w}_{a}}\left[1+\left(\frac{\partial q_{\mathrm{w}_{a}}}{\partial P_{\mathrm{o}_{a}}}\right)^{n} / \sum_{\text {lay }=1}^{M}\left(\frac{\partial q_{\mathrm{w}_{a}}}{\partial P_{\mathrm{wf}_{(\mathrm{seg})}}}\right)^{n}\right]
\end{aligned}
$$




$$
\begin{aligned}
& A_{S_{\mathrm{w}}}^{\mathrm{w}}=\frac{\partial R_{\mathrm{w}_{a}}}{\partial S_{\mathrm{w}_{a}}}=\frac{V_{a} \phi}{\Delta t B_{\mathrm{w}}}-\frac{\partial\left\{\sum_{i=1}^{b}\left[T_{\mathrm{w}_{a i}}\left(\Delta P-\gamma_{\mathrm{w}} \Delta Z\right)\right]^{n+1}\right\}}{\partial S_{\mathrm{w}_{a}}} \\
& -\frac{\partial \mathrm{WI}_{\mathrm{w}_{a}}}{\partial S_{\mathrm{w}_{a}}}\left\{\begin{array}{c}
P_{\mathrm{wf}_{(\mathrm{seg})}}^{n}+\xi_{\mathrm{w}}\left(H_{a}-H_{(\mathrm{seg})}\right)-P_{\mathrm{o}_{a}}^{n+1}-\lambda \frac{L_{a}}{2} \\
\sum_{\text {lay }=1}^{M}\left[\begin{array}{c}
\left.\left(\frac{\partial q_{\mathrm{w}_{a}}}{\partial S_{\mathrm{w}_{a}}}\right)^{n}\left(S_{\mathrm{w}_{a}}^{n+1}-S_{\mathrm{w}_{a}}^{n}\right)+\right] \\
\left(\frac{\partial q_{\mathrm{w}_{a}}}{\partial P_{\mathrm{o}_{a}}}\right)^{n}\left(P_{\mathrm{o}_{a}}^{n+1}-P_{\mathrm{o}_{a}}^{n}\right)
\end{array}\right] \\
\sum_{\text {lay }=1}^{M}\left(\frac{\partial q_{\mathrm{w}_{a}}}{\partial P_{\mathrm{wf}_{(\mathrm{seg})}}}\right)^{n}
\end{array}\right\} \\
& +\mathrm{WI}_{\mathrm{w}_{a}}\left(\frac{\partial q_{\mathrm{w}_{a}}}{\partial S_{\mathrm{w}_{a}}}\right)^{n} / \sum_{\text {lay }=1}^{M}\left(\frac{\partial q_{\mathrm{w}_{a}}}{\partial P_{\mathrm{wf}_{(\mathrm{seg})}}}\right)^{n}
\end{aligned}
$$

The coefficient of the grid with LSWI becomes more complex as indicated in Eqs. 10 and 11, so every submatrix can be regarded as an integral term when the overall numerical model is solved by block pretreatment of generalized minimal residual algorithm (Baohua et al. 2013).

\section{Model validation}

The model proposed in this paper is validated by the test data from a LSWI well (X6-3-321) located in the middle part of the sixth Oilfield in Xing Shugang, and the results of injected water ratio calculated by the model are compared to that of injectivity test data and conventional heavy oil model without LSWI, as shown in Fig. 1.

Figure 1a is the comparative results in 2002, when the LSWI well is divided into four segments by three packers, and Fig. 1b is the comparative results in 2010, when the well is divided into six segments by five packers. It is obviously that conventional heavy oil model cannot simulate the effect of LSWI, but the LSWI model acquires a more accurate injected water ratio close to the injectivity test data in these 2 years.

\section{Case study}

\section{Geological mechanism model}

Considering the specific situation of LSWI wells, reservoir geology and production schemes of the middle part of the sixth Oilfield in Xing Shugang, three mechanism models of multi-layer heterogeneous reservoir were constructed to simulate the LSWI. Each of the models has dimensions of
$820 \mathrm{ft} \times 820 \mathrm{ft} \times 88 \mathrm{ft}$, and is discretized equally by $50 \times 50$ grids horizontally and 54 grids vertically, so it has 135,000 regular grids totally. Generally, there are two kinds of oil layers in the model, and they are high-permeability (100-1000 mD) and interactive low-permeability (5-10 mD) oil layers, which have 22 oil layers totally. One of them is thick high-permeability oil layer (32.8 ft), and 21 of them are interactive low-permeability oil layers, which contain low-permeability oil layers connected with high permeability parts.

As shown in Fig. 2, there are four kinds of interactive low-permeability oil layers in this model from top to bottom: continuous delta front sheet sand oil layers (Fig. 2a: continuous 1-7), partial delta front sheet sand oil layers (Fig. 2b: partial 1-6), delta sheet sand edge oil layers (Fig. 2c: sheet 1-5) and overflow channel sand oil layers (Fig. 2d: overflow 1-3). Especially, sheet 3, continuous 7 and overflow 13 are three independent low-permeability oil layers.

\section{Recovery and vertical sweep efficiency}

Six LSWI projects are designed for the three geological mechanism models, and they are totally developed for 20 years with the same injection water rate. In the initial 5 years of production, five-spot waterflood well pattern is used for the development, and the injector with $30 \mathrm{~m}^{3} /$ day injection water rate is located at the center of the model. Afterwards, the injector is divided into four segments for waterflooding. 5 years later, it is subdivided into 5-10 segments with LSWI by subdivision and restructuring of original segments, and four producers are added into this waterflood well pattern to enhance the. The injection water rate $\left(100 \mathrm{~m}^{3} /\right.$ day $)$ and bottom wellbore pressure of producer keep constant during the 10 years development years with LSWI.

Figures 3, 4 shows the ultimate recovery and vertical sweep efficiency of interactive low-permeability oil layers varying with segment numbers under different modes and rhythm characteristics after developing 20 years. However, it is very difficult to find the regularity between the ultimate recovery and segment numbers, because there are too many factors influencing the ultimate recovery, which include geological models, production schemes and so on. There is no point to analyse the optimum number of intervals under a special model and production scheme. For example, the ultimate recovery under seven segments is higher than that under 8 segments, even the wellbore and oil layers have been subdivided to be much leaner. Presently, some possible instructions can be drawn by us as follows in a broader sense.

The ultimate recovery of model with different rhythms increases at first, and then become smooth, however, the 


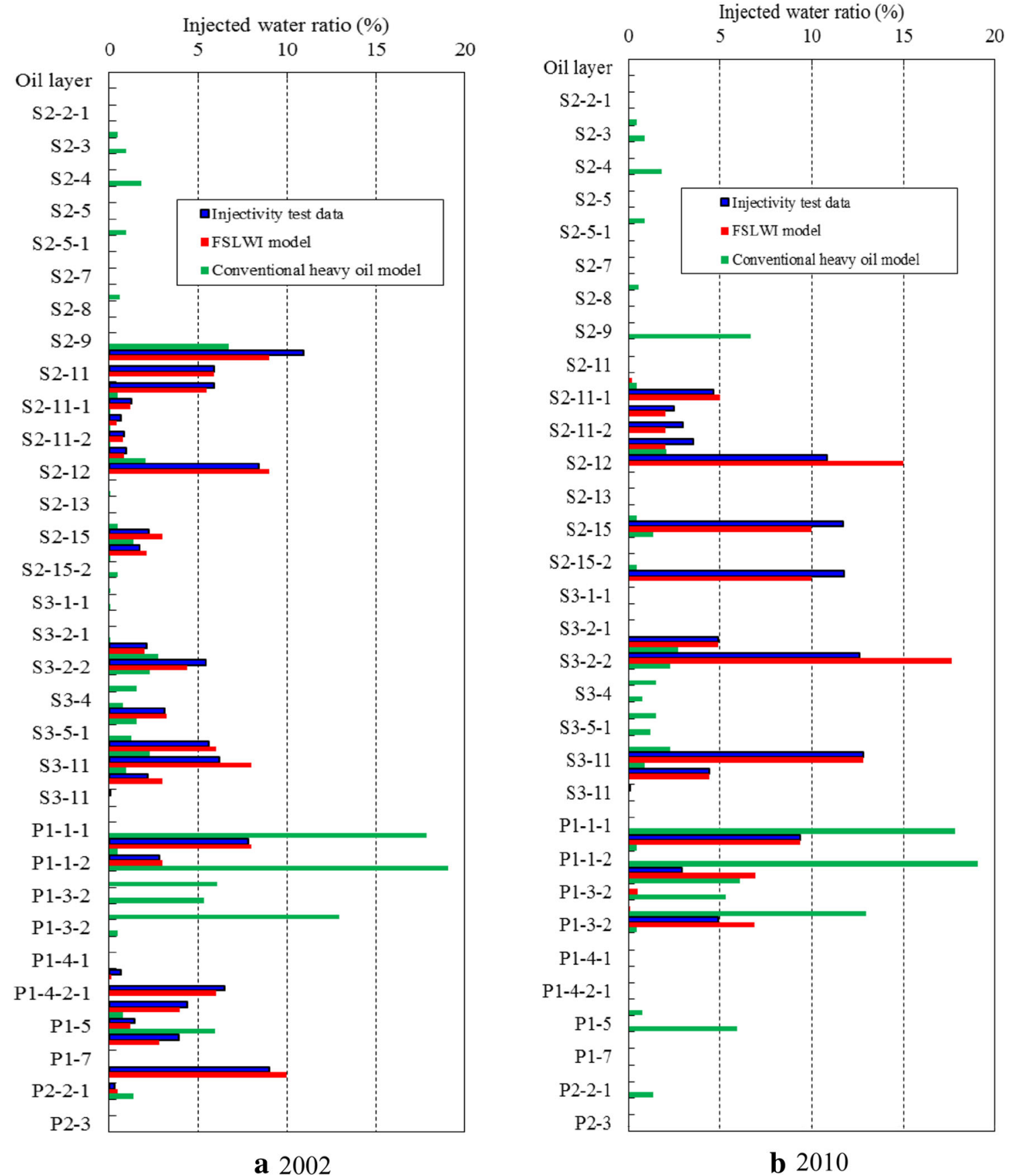

Fig. 1 The comparative results of injectivity test data, LSWI model and conventional heavy oil model

vertical sweep efficiency increases gradually. When segments number of LSWI increases under subdivision of original segments, ultimate recovery and vertical sweep efficiency both increases with strong fluctuation. However, ultimate recovery increases with smaller fluctuation under segmentation and restructuring, and the vertical sweep efficiency increases gradually. It illustrates that more segments and more complete restructuring of segments may not be effected, so the modes of segmentation and segment numbers need to be optimized.

The variation tendency of recovery and vertical sweep efficiency are the same, and high ultimate recovery will be obtained with high vertical sweep efficiency of interactive low-permeability oil layers, so the key of enhancing production lies in increasing vertical sweep efficiency. 


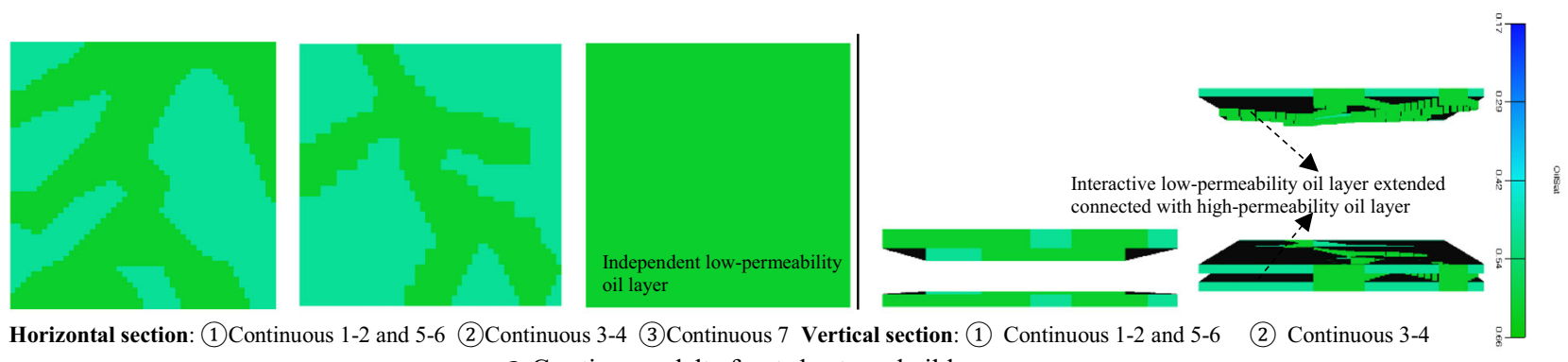

a Continuous delta front sheet sand oil layer

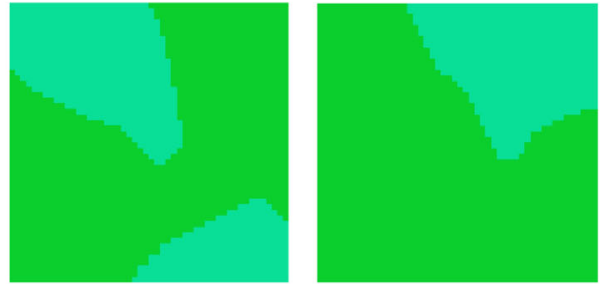

Horizontal section: (1)Partial 1-2 (2) Partial 3-4

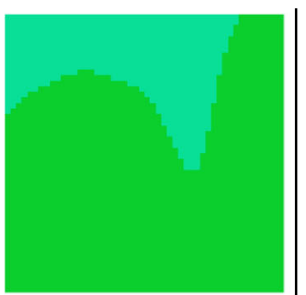

(3) Partial 5-6 Vertical section: (1)Partial 1-2

b Partial delta front sheet sand oil layer
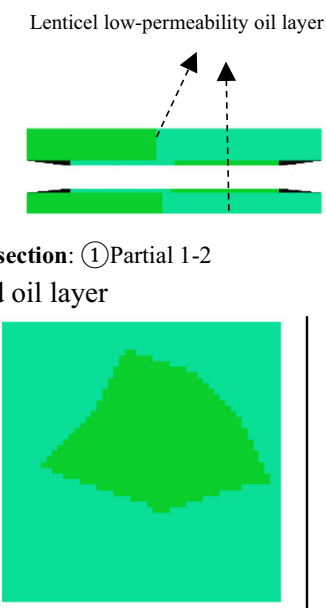

(4) Overflow 4

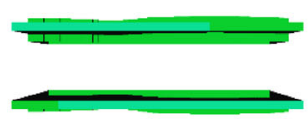

(2) Partial 3-6

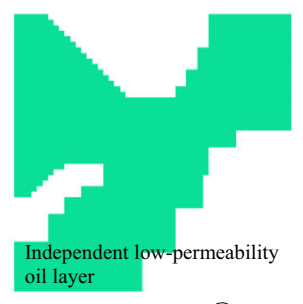

Horizontal section: (1)Overflow 1 (2)Thick high-permeability oil layer (3)Overflow 2 high-permeability oil layer

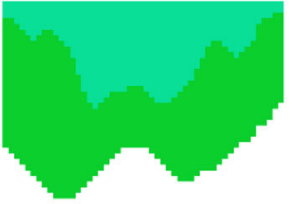

Horizontal section: (1) Sheet 1-2
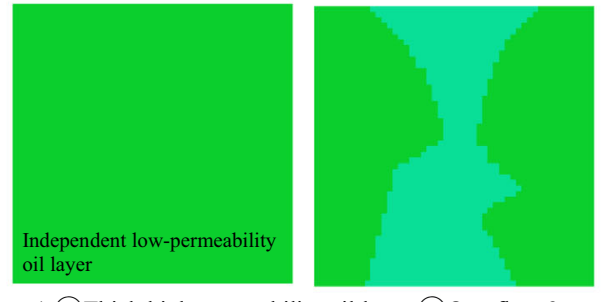

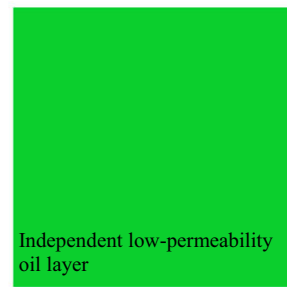

(2) Sheet 3

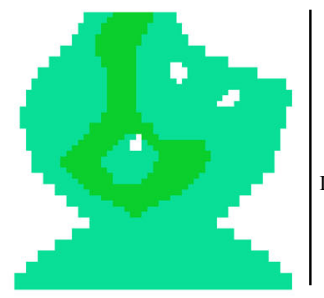

(3) Sheet 4-5

c Overflow channel sand oil layer

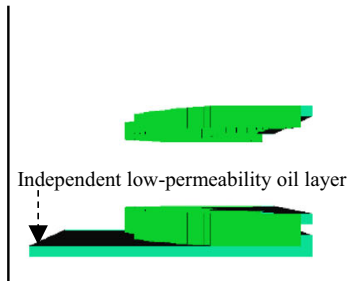

Vertical section: (1) Sheet 1-3

d Edge delta stable sheet sand oil layer

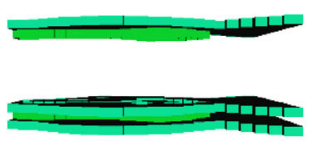

(2) Sheet 4-5

Fig. 2 Initial oil saturation of four types of model with interactive low-permeability reservoirs and high-permeability reservoirs

Rhythm characteristics of high-permeability oil layers have little influence for ultimate recovery of interactive low-permeability oil layers, but segmentation modes have more obvious influence. However, vertical sweep efficiency are almost equal for models with reverse rhythm and composite rhythm, and both higher than positive rhythm model.

\section{Distribution regularity of remaining oil}

Remaining oil caused by incomplete waterflood well pattern cannot be swept effectively. Take the overflow two oil layer shown in Fig. 5 as an example, only one injector is located in the center of the interactive oil layer, and its lowpermeability part connects with high-permeability parts at both sides, so there is no producer corresponded with the injector in low-permeability parts. Consequently, most remaining oil concentrates in I and II parts. Besides, although there is no injector corresponded with producer in these two high permeability edge parts, water injected for low-permeability parts flows to these parts, so remaining oil is only trapped in top and bottom corners of these parts.

Because of the vertical heterogeneity of the multi-layer reservoir models, the injected water mainly flow into the thick high-permeability oil layers rapidly, though these layers have reached extreme high water content, so the 
Fig. 3 The ultimate recovery varying with segment numbers under different modes
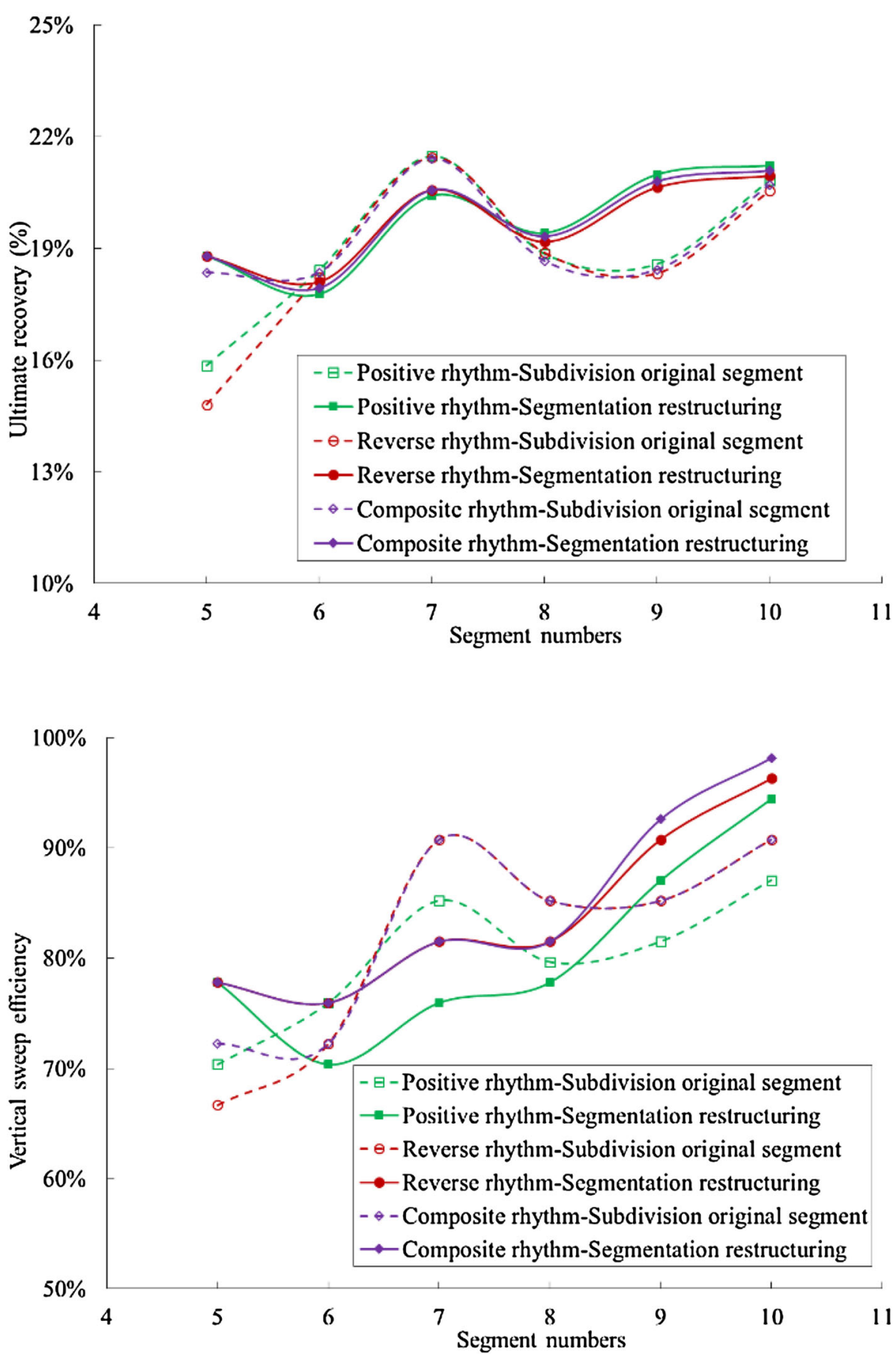

adjacent low-permeability oil layers have not been swept effectively. As Fig. 6 shows, overflow 1 and 2, which are the upper and lower oil layers of the thick high-permeability oil layer, have not been swept when the LSWI well are divided into five segments. That is because bottom wellbore pressure of injector is less than threshold pressure, so water cannot be injected in these low-permeability oil layers.
Independent low-permeability oil layers have poor physical properties, so they are difficult to be swept even by LSWI. As shown in Fig. 7, sheet 3 is independent low-permeability oil layer, which cannot be swept when the injector is divided into five segments by LSWI. After subdividing into ten segments, the oil layer can be swept finally. However, the waterflood production isn't entirely effective, so more stimulating 


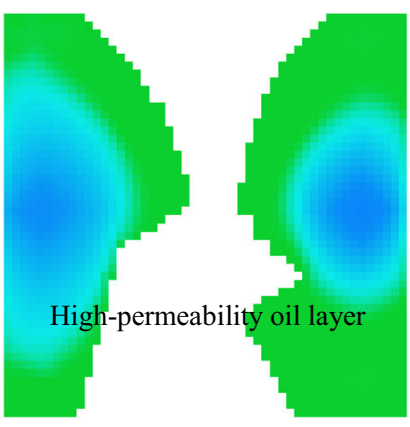

(Top)

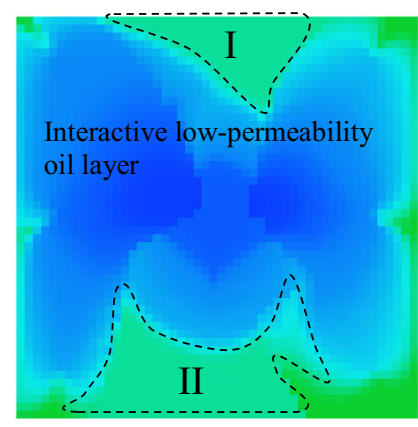

(Middle)
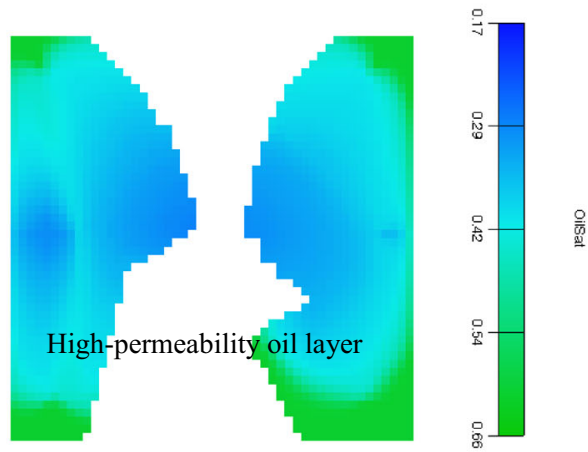

(Bottom)

Fig. 5 Oil saturation distribution of overflow 2 oil layer after developing 20 years with ten segments

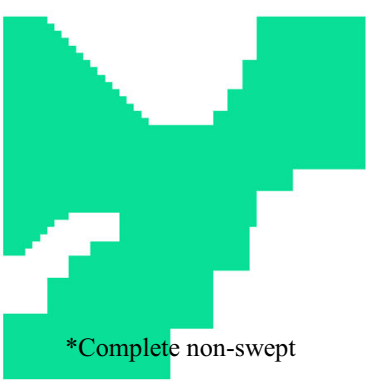

(Overflow 1)

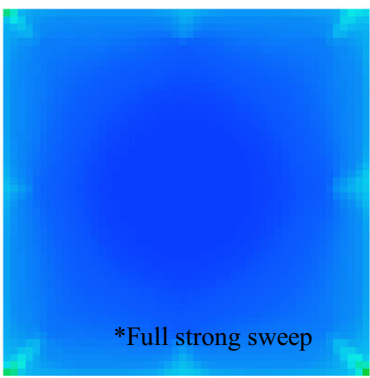

(Thick high-permeability oil layer)

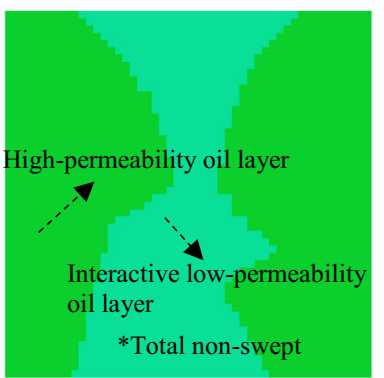

(Overflow 2)

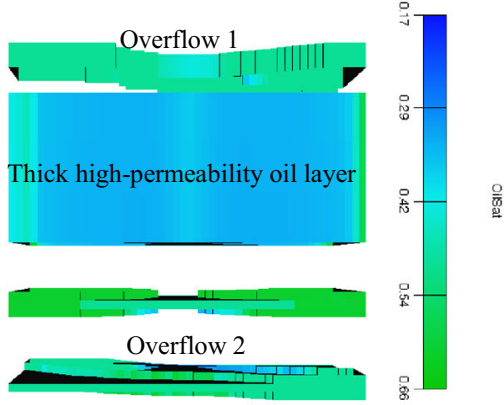

(Vertical section of 3 oil layers before)

Fig. 6 Oil saturation distribution of overflow 1, 2 and high permeability oil layers after developing 20 years with five segments

Fig. 7 Oil saturation distribution of Sheet 3 oil layer after developing 20 years with five and ten segments

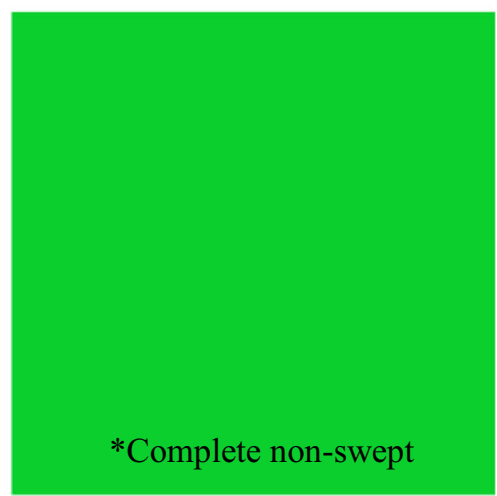

(5 segments)

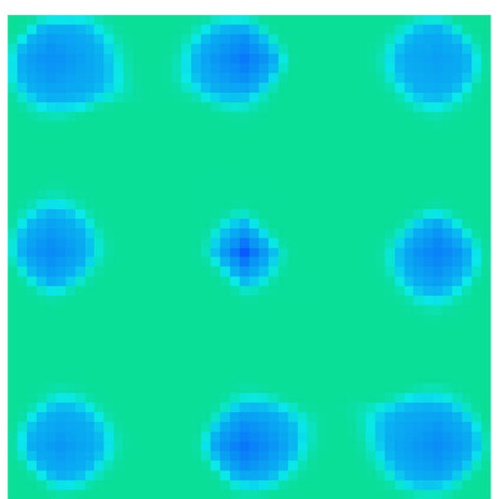

(10 segments)

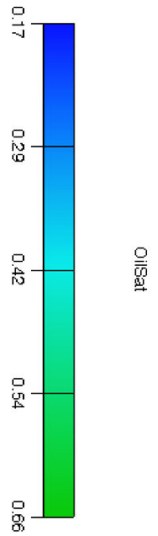

solved implicitly, and then well model of LSWI is established, which coupled with oil-water two phases model to form the final numerical models. It can also be represented as close linear system with seven diagonal sparse coefficient matrix, and solved by block pretreatment of generalized minimal residual algorithm. According to the theory of LSWI, the numerical model proposed, reservoir stratum distribution and injector segments characterized in the Chinese Daqing Oilfield, three multi-layer reservoir
Injection pressure adjusted by water chokes, well grid pressure and fluid saturation are made as three unknown parameters, and the source terms of injector wellbore are measures are necessary, such as hydraulic fracturing and gas injection.

\section{Conclusions}


mechanism models are established with positive rhythm, negative rhythm and composite rhythm, respectively in high-permeability oil layers.

Low-permeability oil layers are primary potential for multi-layer reservoirs with extreme high water content, and LSWI will help them to get higher recovery. More segments is not the goal of LSWI, the structure of wellbore segments need to correspond with distribution of remaining oil, and vertical heterogeneity should be weakened. The key of enhancing recovery is to increase vertical sweep efficiency, the remaining oil caused by vertical heterogeneity and poor physical properties can be waterflooded by adjusting structure of segmentation. However, some other stimulating measures are necessary to be used for developing the remaining oil caused by bad connectivity between injectors and producers.

Acknowledgments This study has been carried out under the framework of the national mega project of science research (2011ZX05010-002) financed by Chinese government, and has been partially supported by Petro-china.

Open Access This article is distributed under the terms of the Creative Commons Attribution 4.0 International License (http:// creativecommons.org/licenses/by/4.0/), which permits unrestricted use, distribution, and reproduction in any medium, provided you give appropriate credit to the original author(s) and the source, provide a link to the Creative Commons license, and indicate if changes were made.

\section{References}

Baohua W, Shuhong W, Dakuang H et al (2013) Block compressed storage and computation in large-scale reservoir simulation. Pet Explor Dev 40(4):462-465
Dakuang H (2010) Discussions on concepts, countermeasures and technical routes for the redevelopment of high water-cut oilfields. Pet Explor Dev 37(5):583-591

Ding DY (2011) Coupled simulation of near-wellbore and reservoir models. J Pet Sci Eng 76:21-36

Dumkwu FA et al (2012) Review of well models and assessment of their impacts on numerical reservoir simulation performance. J Pet Sci Eng 15:174-186

Gao D, Ye J, Hu Y et al (2015) Numerical reservoir simulation constrained to fine separated layer water injection. Chin $\mathbf{J}$ Comput Phys 32(3):38-44

He L, Xiaoguang P, Kai L (2013) Current status and trend of separated layer water flooding in China. Pet Explor Dev 40(6):733-737

Jigen Y, Xianghong W, Yixiang Z et al (2007) Study on computer assisted history-matching method in corner point grids. Acta Petrolei Sinica 28(2):83-86

Lin CY, Huang LH, Li DL et al (2011) The coupling study of throttle and reservoir seepage of water injection in development of oil field. Chin J Comput Mech 28(4):607-609

Stone TW et al (1989) A comprehensive wellbore/reservoir simulator. SPE 18419

Villanueva-Triana B et al (2013) Rigorous simulation of production from commingled multilayer reservoirs under various crossflow and boundary conditions. SPE 164501

Yan Z, Xie J, Yang W et al (2008) Method for improving history matching precision of reservoir numerical simulation. Pet Explor Dev 35(2):225-227

Zhao G, Sun W, He X (2012) Reservoir simulation model based on stratified water injection and its application. J Northeast Pet Univ 36(6):82-87 